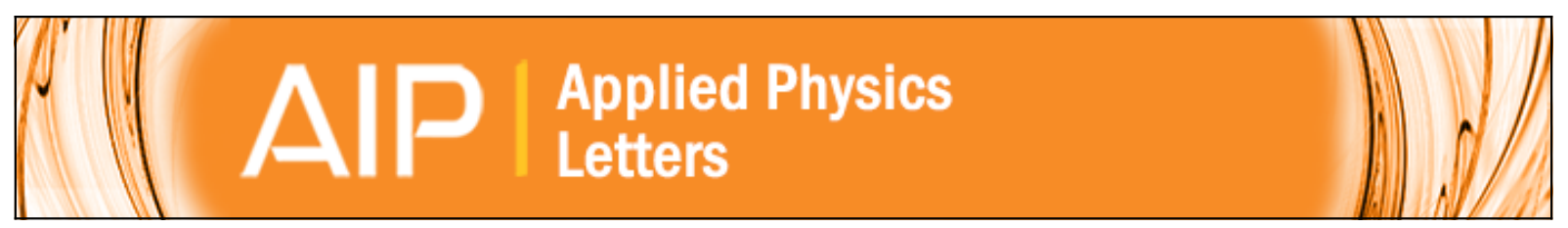

\title{
Spin wave spectra in perpendicularly magnetized permalloy rings
}

X. Zhou, J. Ding, M. Kostylev, and A. O. Adeyeye

Citation: Applied Physics Letters 106, 112403 (2015); doi: 10.1063/1.4916029

View online: http://dx.doi.org/10.1063/1.4916029

View Table of Contents: http://scitation.aip.org/content/aip/journal/apl/106/11?ver=pdfcov

Published by the AIP Publishing

\section{Articles you may be interested in}

Stability of standing spin wave in permalloy thin film studied by anisotropic magnetoresistance effect

J. Appl. Phys. 118, 233909 (2015); 10.1063/1.4937918

Reciprocal Damon-Eshbach-type spin wave excitation in a magnonic crystal due to tunable magnetic symmetry Appl. Phys. Lett. 102, 012403 (2013); 10.1063/1.4773522

Spin wave resonance excitation in ferromagnetic films using planar waveguide structures J. Appl. Phys. 108, 023907 (2010); 10.1063/1.3435318

High-intensity Brillouin light scattering by spin waves in a permalloy film under microwave resonance pumping J. Appl. Phys. 102, 103905 (2007); 10.1063/1.2815673

Multiple ferromagnetic resonance in mesoscopic permalloy rings

J. Appl. Phys. 97, 10A712 (2005); 10.1063/1.1851932

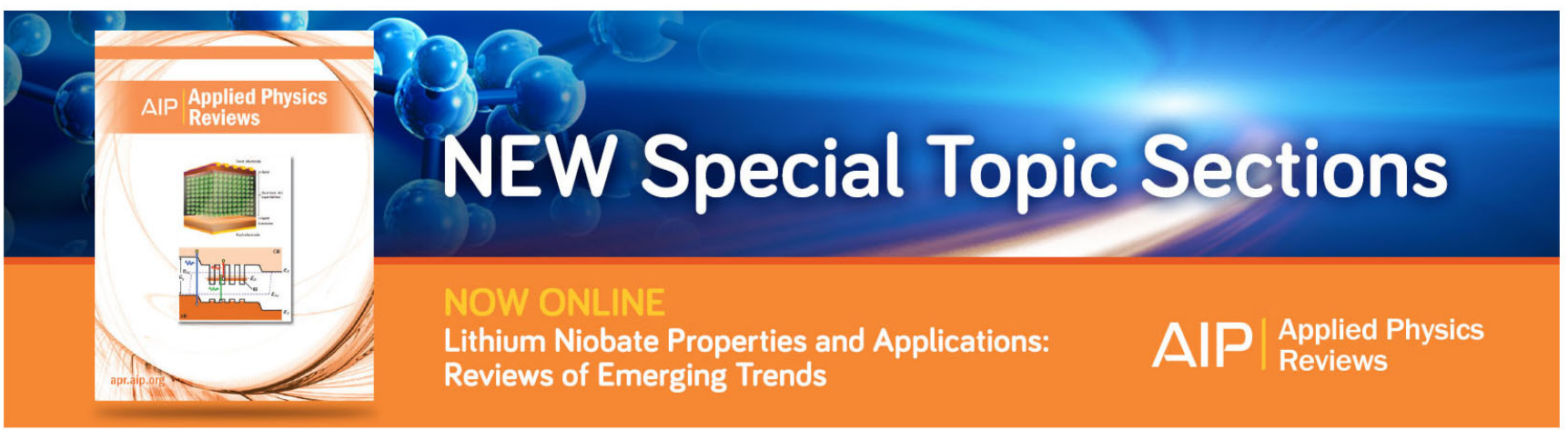




\title{
Spin wave spectra in perpendicularly magnetized permalloy rings
}

\author{
X. Zhou, ${ }^{1}$ J. Ding, ${ }^{1}$ M. Kostylev, ${ }^{2}$ and A. O. Adeyeye ${ }^{1, a)}$ \\ ${ }^{1}$ Information Storage Materials Laboratory, Department of Electrical and Computer Engineering, \\ National University of Singapore, Singapore 117576 \\ ${ }^{2}$ School of Physics, University of Western Australia, Crawley, Western Australia 6009, Australia
}

(Received 23 January 2015; accepted 11 March 2015; published online 20 March 2015)

\begin{abstract}
The dynamic behavior of perpendicularly magnetized permalloy circular rings is systematically investigated as a function of film thickness using broadband field modulated ferromagnetic resonance spectroscopy. We observed the splitting of one spin wave mode into a family of dense resonance peaks for the rings, which is markedly different from the single mode observed for continuous films of the same thickness. As the excitation frequency is increased, the mode family observed for the rings gradually converges into one mode. With the increase in the film thickness, a sparser spectrum of modes is observed. Our experimental results are in qualitative agreement with the dynamic micromagnetic simulations. (C) 2015 AIP Publishing LLC.

[http://dx.doi.org/10.1063/1.4916029]
\end{abstract}

The dynamic properties of in-plane magnetized patterned nanostructures, such as ferromagnetic dots, ${ }^{1-3}$ rings, ${ }^{4-6}$ and wires ${ }^{7-9}$ have been extensively investigated due to their potential in applications such as microwave filters, ${ }^{10}$ smart sensors, ${ }^{11,12}$ and other high performance microwave and spintronic devices. ${ }^{13,14}$ Recently, there has been an increasing research interest on fundamental understanding of the dynamic properties of perpendicularly magnetized dot arrays using ferromagnetic resonance (FMR) spectroscopy. ${ }^{15,16}$ With the field applied out of plane, high in-plane symmetry is preserved, and therefore, it is easier to interpret theoretically with regards to the observed spin wave (SW) modes. ${ }^{17}$

To date, multiple spin wave modes were observed in perpendicularly magnetized submicron dot arrays in saturated field range. ${ }^{16-18}$ Dipole-exchange theory of in-plane spin wave spectra ${ }^{17}$ has been utilized to explain this phenomenon. It shows that a high static magnetic field is necessary to excite high frequency SWs in dots. Ferromagnetic rings represent a natural extension of the dots geometry, in which the central part is removed. It might be a candidate to scale down the resonance field compared to the disks of the same dimension due to its shape anisotropy along the radial direction. This provides additional freedom to control the spin wave spectra. In order to reduce the resonance field for high frequency SWs, it is imperative to investigate the dynamic properties of perpendicularly magnetized circular rings.

In this work, we present a systematic study on the spin wave excitation of perpendicularly magnetized mesoscopic circular ring arrays as a function of frequency and film thickness. The FMR spectra of the rings are markedly different from the corresponding continuous films deposited under similar conditions. For rings with a fixed film thickness, a drastic modification in the resonance field and mode numbers of the FMR spectrum is observed for different excitation frequencies. Additionally, for the same excitation frequency,

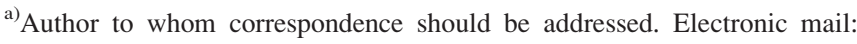
eleaao@nus.edu.sg
}

the resonance field of permalloy rings is remarkably reduced when the film thickness is increased.

The periodic arrays of isolated $\mathrm{Ni}_{80} \mathrm{Fe}_{20}$ (permalloy) rings were fabricated on a silicon substrate using deep ultraviolet lithography (DUV) at $248 \mathrm{~nm}$ exposure wavelength followed by electron beam evaporation and ultrasonic assisted lift off process in OK73 resist thinner. Permalloy rings with thickness in the range from $10 \mathrm{~nm}$ to $80 \mathrm{~nm}$ were deposited at a constant rate of $0.2 \AA / s$ with a base pressure of $2 \times 10^{-8}$ Torr. Details of the fabrication process were described elsewhere. ${ }^{19}$ Scanning electron microscope (SEM) was used to verify the dimensions of the rings. The inset of Fig. 1(a) is the corresponding SEM image of the $400 \mathrm{~nm}$ wide circular ring with an outer diameter of $3 \mu \mathrm{m}$.

The FMR spectra were characterized using broadband field modulated FMR setup at room temperature. Different from a vector network analyzer (VNA) - FMR setup, the microwave signal was generated by a continuous wave (c.w.) microwave generator at a specific frequency in the range from $6 \mathrm{GHz}$ to $17 \mathrm{GHz}$. The sample was positioned on top of a $50 \Omega$ microstrip board, with the ring arrays facing the board, similar to the technique reported in Ref. 20. A static magnetic field in the range from $18 \mathrm{kOe}$ to 0 and an AC modulating field of \pm 20 Oe has been applied perpendicular to the film plane (defined as $\mathrm{z}$ direction). An interferometric device and applied-field modulation were used to detect the FMR signal with an ultra-low signal to noise ratio. ${ }^{21}$ The device "core" is similar to the recently described simple arrangement from Ref. 22. Extra stages not present in Ref. 22 further decrease the noise level and make the device a stand-alone instrument allowing taking measurements without making use of a vector network analyzer. The output dc signal of the interferometric device is fed into a digital lock-in amplifier locked to the field modulation signal in order to further increase the signal to noise ratio. The FMR signal detected in this way represents the first derivative of the field-sweeping absorption curve at a selected frequency.

Fig. 1(b) shows the normalized FMR spectra taken on the permalloy continuous film and permalloy ring arrays at 

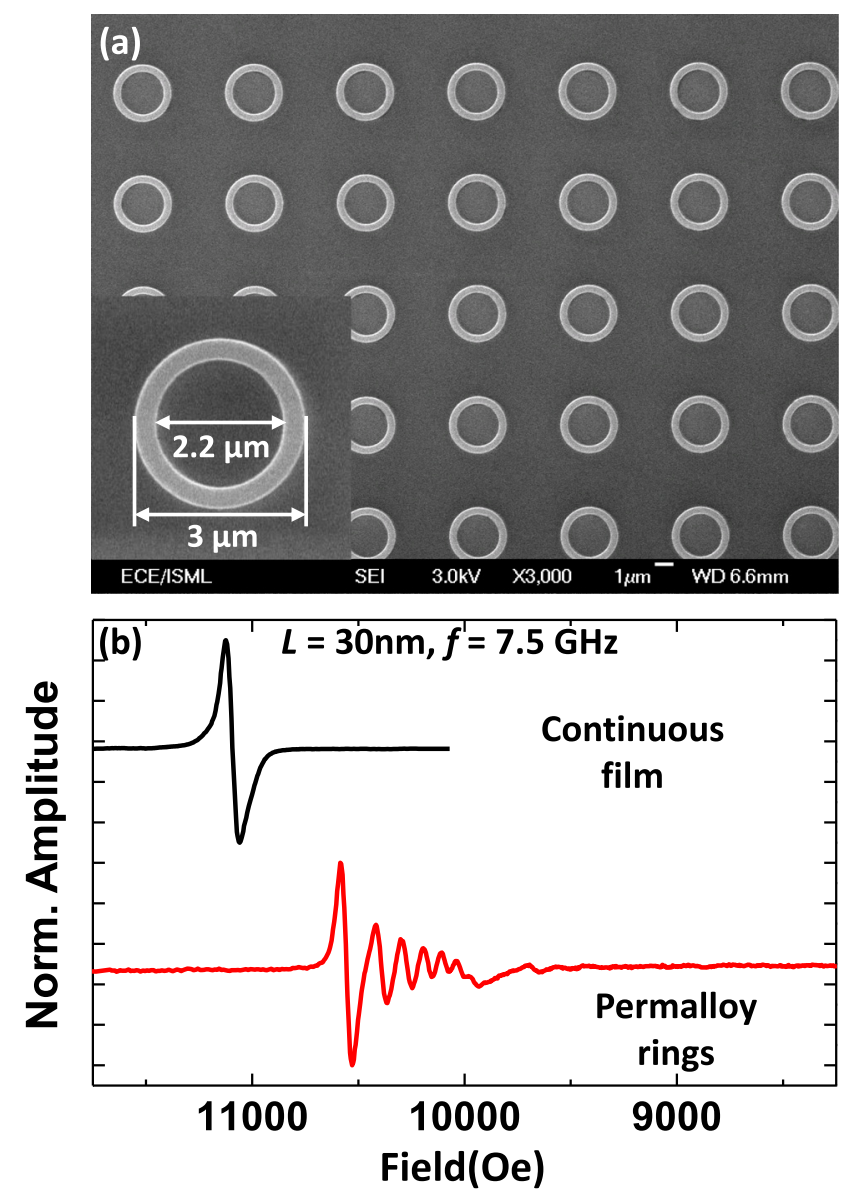

FIG. 1. (a) Scanning electron micrograph of wide permalloy circular rings with outer diameter of $3 \mu \mathrm{m}$ and width of $400 \mathrm{~nm}$; (b) experimental FMR spectra at $7.5 \mathrm{GHz}$ for permalloy rings and continuous film with the thickness of $30 \mathrm{~nm}$.

$f=7.5 \mathrm{GHz}$ for the film thickness of $30 \mathrm{~nm}$. We observed that the resonance field is significantly reduced in the permalloy rings compared with the continuous film. For the continuous film, only a single spin wave mode was observed, while multiple peaks were clearly visible for permalloy circular rings in the field range larger than $10 \mathrm{kOe}$. The additional resonance peaks for permalloy rings appear in the field region below the fundamental mode and the amplitude decreases as the magnetic field is reduced. The hysteresis loop (not presented in the paper) shows that permalloy rings with a thickness of $30 \mathrm{~nm}$ are fully saturated when the amplitude of the applied magnetic field is higher than $9 \mathrm{kOe}$.

Fig. 2(a) shows the FMR spectra at various selected frequencies for $30 \mathrm{~nm}$ thick permalloy rings and continuous film. As the frequency is increased, the resonance field of both the permalloy rings and continuous film increases. Since both the dispersion relations for continuous film and the fundamental mode of permalloy rings follow the Kittel equation, ${ }^{18,23}$ this can be explained by the decrease of the internal field when the applied field is reduced. For all the frequencies, the resonance field of continuous film is higher than that of permalloy rings, due to the highest demagnetizing factor along $\mathrm{Z}$ direction $(=1)$ for continuous film. The results of rings also show that the higher order modes are merging with the main peak with the increase in frequency and only the fundamental mode remains when the frequency is higher than $14 \mathrm{GHz}$ (although there is a visible extra peak at $f=14 \mathrm{GHz}$, it is not pronounced). The FMR spectrum for permalloy rings is similar to that of continuous film when $f=16 \mathrm{GHz}$. To further investigate how the modes change with frequency, the resonance fields of different modes were extracted as a function of frequency, as displayed in Fig. 2(b). As we can see from the figure, there are eight modes at $f=6.5 \mathrm{GHz}$, and the field difference between the fundamental mode and the eighth mode is around $1 \mathrm{kOe}$. With the increase of frequency, the field gaps between neighboring modes are smaller.

Presented in Fig. 3(a) are the FMR spectra taken at $f=8 \mathrm{GHz}$ for the rings with thicknesses of $20 \mathrm{~nm}, 30 \mathrm{~nm}$, and $60 \mathrm{~nm}$. The resonance field decreases with the increase in the film thickness. A sparser FMR spectrum is observed for the thicker rings. To get a comprehensive understanding on how FMR spectra are tailored by controlling the thickness of the rings, resonance fields of the fundamental mode and the second mode for rings with the thicknesses of $20 \mathrm{~nm}$, $30 \mathrm{~nm}$, and $60 \mathrm{~nm}$ were first extracted as a function of frequency. As shown in Fig. 3(b), for $L=60 \mathrm{~nm}$, we observed that the second mode still exists at higher frequencies (14 $\mathrm{GHz}-16 \mathrm{GHz}$ ), compared with $L=20 \mathrm{~nm}$, while there is only

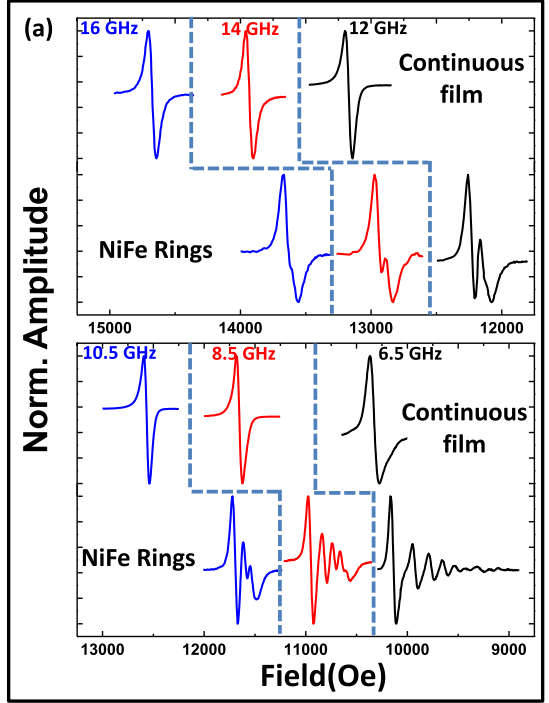

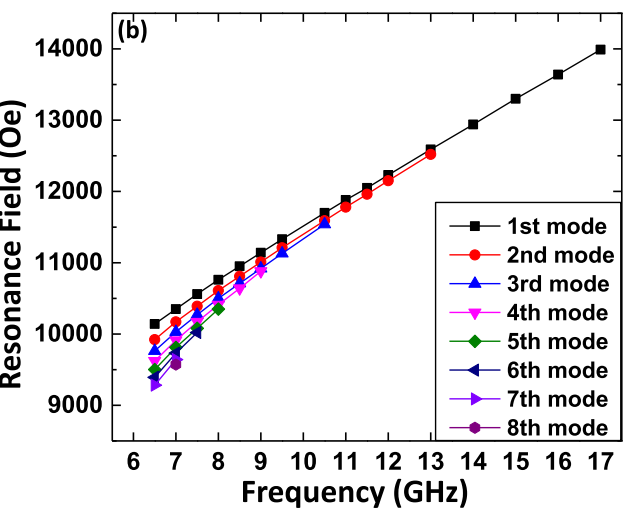

FIG. 2. (a) Experimental FMR spectra for $30 \mathrm{~nm}$ thick permalloy rings and continuous film at different frequencies $(f=6.5 \mathrm{GHz}, \quad 8.5 \mathrm{GHz}, \quad 10.5 \mathrm{GHz}$, $12 \mathrm{GHz}, 14 \mathrm{GHz}$, and $16 \mathrm{GHz}$ ); (b) resonance fields as a function of frequency for $30 \mathrm{~nm}$ thick permalloy rings. 

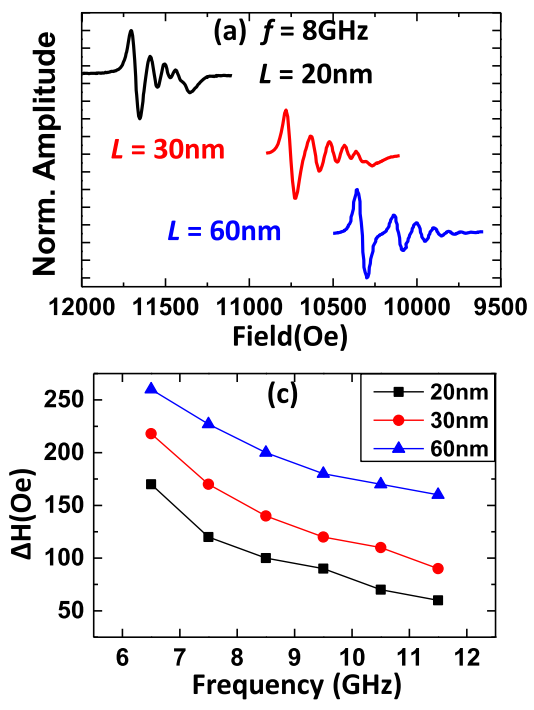

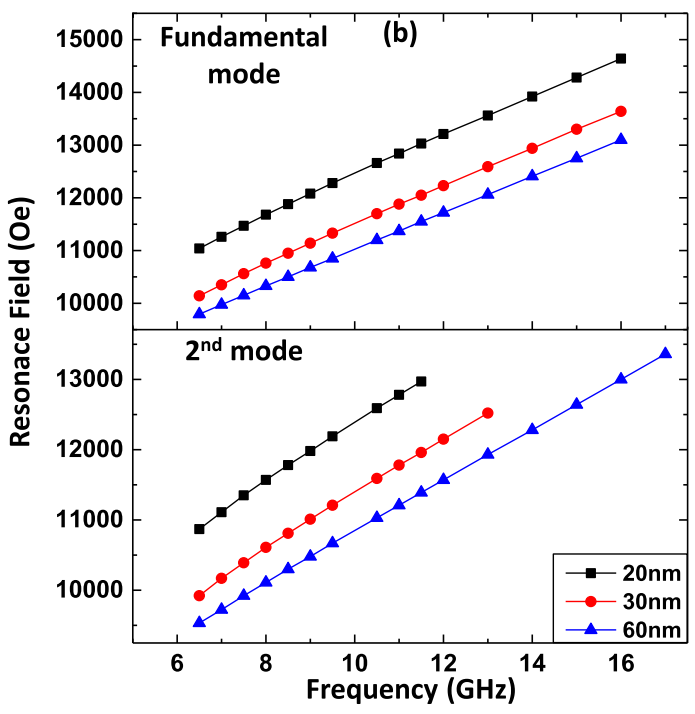

FIG. 3. (a) Experimental FMR spectra at $8 \mathrm{GHz}$ for permalloy rings with the thickness of $20 \mathrm{~nm}, 30 \mathrm{~nm}$, and $60 \mathrm{~nm}$; (b) resonance fields of the fundamental mode and the second mode (shown separately) as a function of frequency for $20 \mathrm{~nm}, 30 \mathrm{~nm}$, and $60 \mathrm{~nm}$ thick permalloy rings; (c) field gap between the fundamental mode and the second mode as a function of frequency for permalloy rings with the thickness of $20 \mathrm{~nm}, 30 \mathrm{~nm}$, and $60 \mathrm{~nm}$. one mode left at $11.5 \mathrm{GHz}$. Similar to the results shown in Fig. 3(a), more modes are observed for thicker rings. It is also shown that for both the fundamental and the second modes, the resonance field increases with the increase of frequency. At a fixed frequency, the resonance field of $L=60 \mathrm{~nm}$ shifts downwards to a smaller value for both modes compared with $L=20 \mathrm{~nm}$ and $30 \mathrm{~nm}$. For the fundamental mode, as the thickness of the rings increases, the demagnetizing factor along $\mathrm{z}$ direction decreases, which means that the internal field is larger. According to Kittel equation, for the same frequency, resonance field for thicker permalloy rings is lower. Fig. 3(c) presents the field gap between the fundamental mode and the second mode extracted as a function of frequency for all the three thicknesses. It can be clearly seen that the field gap increases with thickness. This will be discussed in detail later.

In order to understand how frequency and thickness affect the FMR spectra, we performed dynamic micromagnetic simulation with the LLG micromagnetic simulator. Standard parameters for permalloy (gyromagnetic ratio $\gamma=2.8 \mathrm{GHz} / \mathrm{kOe}$, saturation magnetization $M_{s}=810 \mathrm{emu} \cdot \mathrm{cm}^{-3}$, exchange constant $A=1.30 \times 10^{-6} \mathrm{erg} \cdot \mathrm{cm}^{-1}$, damping constant $\alpha=0.01$, and anisotropy constant $K_{U}=0$ ) were utilized for the simulation. The mask in the simulation was edge corrected and discretized by unit cell size of $10 \mathrm{~nm} \times 10 \mathrm{~nm} \times 10 \mathrm{~nm}$.

Let us first focus on the simulation results for the thickest rings $(L=80 \mathrm{~nm})$. Presented in Fig. 4(a) is the comparison of simulation results on half-ring at the frequency of 9.5 $\mathrm{GHz}$ (black line) and $16 \mathrm{GHz}$ (red line). The figure demonstrates four main resonance peaks at both frequencies corresponding to different radial modes of the half-ring. More importantly, for $f=9.5 \mathrm{GHz}$ the fundamental peak (which is the-highest-field peak) and the next-order one at about 10 kOe in Fig. 4(a) display a fine structure. This fine structure reveals that there is a family of modes which is characterized by a fundamental radial distribution of dynamic magnetization. Fig. 4(b) shows the corresponding mode profiles at each resonance field $(\mathrm{H}=10975 \mathrm{Oe}, 10895 \mathrm{Oe}, 10794 \mathrm{Oe}$, and 10693 Oe $(\mathrm{n}=1) ; 10010$ Oe and 9808 Oe $(\mathrm{n}=2) ; 9005$ Oe $(n=3)$; and 8201 Oe $(n=4))$. We can see from Fig. 4(b) that the extra small peaks near the-highest-field peak (next-order
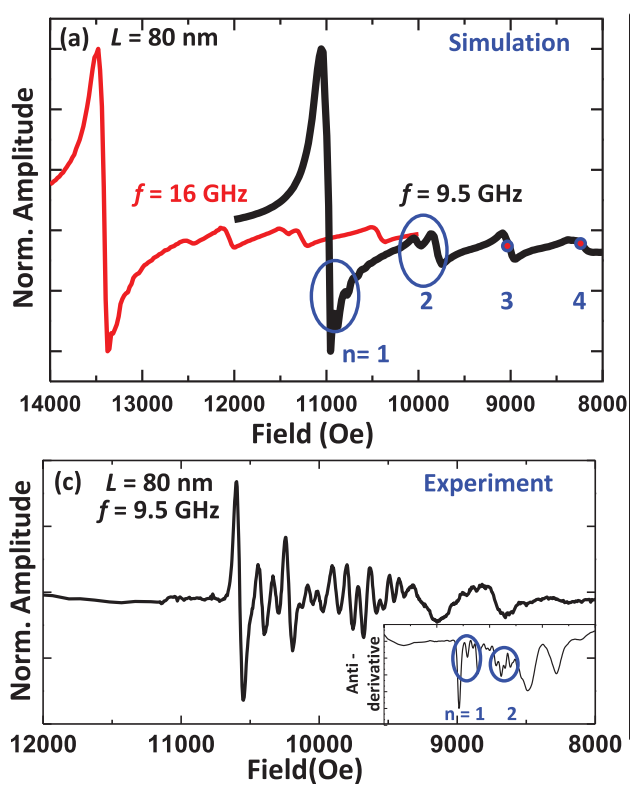
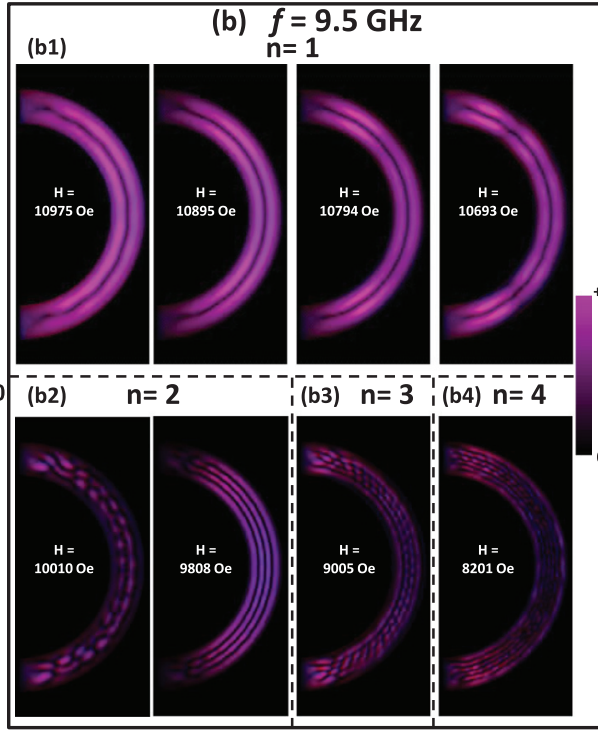

(b3) $n=3$, (b4) $n=4$

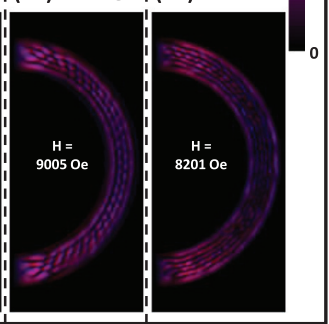

FIG. 4. (a) Simulated FMR spectra for $80 \mathrm{~nm}$ thick permalloy rings at $f=9.5 \mathrm{GHz}$ (Black line) and $16 \mathrm{GHz}$ (Red line); (b) simulated mode profiles for the family of fundamental mode at $\mathrm{H}=10975 \mathrm{Oe}, 10895 \mathrm{Oe}, 10794 \mathrm{Oe}$, and 10693 Oe (b1), the family of second order mode at $\mathrm{H}=10010 \mathrm{Oe}$ and 9808 Oe (b2), and two higher order modes at $\mathrm{H}=9005 \mathrm{Oe}$ and $8201 \mathrm{Oe}$ (b3) and (b4), corresponding to each resonance field at $f=9.5 \mathrm{GHz}$. Color scale bar represents the relative absorption amplitude; (c) corresponding experimental FMR spectrum for $80 \mathrm{~nm}$ thick rings at $f=9.5 \mathrm{GHz}$. The inset in (c) shows the antiderivative of the FMR spectra. 
peak) have the same radial mode with the fundamental peak (next-order peak) but shows azimuthal non-uniformity.

A similar behavior is seen for the next-order mode and may be explained in the same way as a response of a family of modes with the same radial structure. In Fig. 4(c), we present the respective experimental data. The inset shows the anti-derivative of the raw data presented in Fig. 4(c). We speculate that the raw data show evidence of splitting of the fundamental and the next-order modes into families. This is more visible in the inset figure, where the peaks can be divided into two series " 1 " and " 2 ," based on their amplitudes. And in each series the peaks spread in a field range of around 200 Oe. We speculate that "1" corresponds to the fundamental family and " 2 " to the next-order family. Importantly, our simulation result in Fig. 4(a) shows that the fine structure gradually disappears with an increase in the FMR frequency. This effect is similar to what one sees in the experimental Fig. 2(b).

There is, however, a distinct difference between Figs. 4(a) and 4(c). The fine structure is much more resolved in the experimental data and the fine-structure peaks have larger amplitudes. We claim that this may be due to a more pronounced non-uniformity of the magnetization ground state in the experiment with respect to the model. Indeed, the perpendicular-to-plane direction represents a hard axis of the ring shape anisotropy. Hence, this direction is a direction of unstable equilibrium. Therefore, for the applied fields slightly above the saturating field, the static magnetization vector "takes any opportunity" to flip back into the plane locally. This happens, for instance, near the ring edges, where the out-of-plane and in-plane demagnetizing fields are stronger. We claim that this may also happen locally due to spatial non-uniformities of the material parameters (saturation magnetization, ring thickness, etc). The process of establishing magnetic equilibrium is highly nonlinear because it is governed by an intrinsically nonlinear Landau-Lifschitz equation. Therefore, near the point of unstable equilibrium tiniest spatial fluctuations in the material parameters may strongly amplify the tendency of the magnetization vector to flip into the ring plane.

In reality, it may not be a complete flip of the static magnetization vector into the plane, since the exchange interaction will not allow strong variations in the vector direction on the length scale given by the exchange length for permalloy $(5 \mathrm{~nm})$, but small random fluctuations of the angle of static magnetization with the film normal is plausible on the length scale larger than $5 \mathrm{~nm}$. This non-uniformity breaks the symmetry of the modes and some modes which do not couple to the spatially uniform microwave magnetic field for a perfectly uniform material become driven and visible in the FMR spectra.

Similarly, the modes which are characterized by a weak coupling to the microwave driving field because of their natural type of symmetry may get their symmetry further broken, which will lead to an increase in their amplitudes and better resolution of the family of these modes. We believe this is what actually happens in our experiment.

This effect is different from the usual one of the inhomogeneous linewidth broadening. In the latter case, material non-uniformities locally modify resonance frequencies which may lead to a lift of degeneracy of mode frequencies and thus to a resonance linewidth broadening. In the former case, the non-uniformity modifies coupling of the resonance modes to a source of the driving field. Non-degenerate modes of a discrete spectrum which are not driven or driven only weakly for an ideal material become driven or more strongly driven due to the non-uniformities.

Thus, in the case of the inhomogeneous linewidth broadening, the effect of modification of resonance frequency by non-uniformity dominates, but the coupling to the driving field is essentially the same throughout the sample. However, in the case of the effect which we observe in the present work, the non-uniformity delivers negligible contribution to the resonance frequency or field but noticeably affects mode excitation amplitudes. The latter is possible when the mode spectrum is fundamentally discrete, as in our case of micron-size rings.

Our explanation above is consistent with the observation that the fine structure becomes less pronounced with a decrease in the ring thickness and an increase in the FMR frequency. With a decrease in the film thickness, the effective inplane demagnetizing factors ${ }^{24}$ for the resonance modes decrease. This results in a denser spectrum of modes. The field distance between the modes becomes more comparable with the resonance line width and individual peaks within families of modes become less resolved. Furthermore, the families' traces become more compact (less spread in the field space).

With an increase in the frequency, the resonance spectrum shifts upwards in the field. The fundamental and the next-order peak now exist at the fields deeper into the magnetically saturated regime for the rings. The spatial fluctuations of the static vector direction become smaller which translates into a smaller break of mode symmetries and hence in a less resolved fine structure.

In summary, we have conducted a systematic investigation on the dynamic behavior of perpendicularly magnetized permalloy circular rings with different thicknesses. Multiple peaks were observed in the FMR spectra of the ring structures. As the frequency is increased, the resonance field increases and the extra peaks observed at lower frequencies gradually merge into one single mode at higher frequencies. We also found that, with thicker rings, the resonance field is reduced significantly and the FMR spectra become less dense.

Dynamic micromagnetic simulations were used to explain our experimental results. They demonstrated existence of fine structure of resonance peaks. This fact allowed us to suggest explanation of the rich spectrum existing for thicker rings at small resonance frequencies as due to amplification of the response of the modes constituting the fine structure due to spatial fluctuations of the local equilibrium direction for the static magnetization vector. The latter is possible because the direction normal to the ring plane represents the direction of the unstable equilibrium for the vector.

This work was supported by the National Research Foundation under NRF-CRP 10-2012-03, SMF-NUS New Horizon Awards, Ministry of Education of Singapore, the Australian Research Council, the University of Western Australia (UWA), and UWA's Faculty of Science. 
${ }^{1}$ R. Zivieri, F. Montoncello, L. Giovannini, F. Nizzoli, S. Tacchi, M. Madami, G. Gubbiotti, G. Carlotti, and A. O. Adeyeye, Phys. Rev. B 83, 054431 (2011).

${ }^{2}$ G. Gubbiotti, S. Tacchi, M. Madami, G. Carlotti, A. O. Adeyeye, and M. Kostylev, J. Phys. D: Appl. Phys. 43, 264003 (2010).

${ }^{3}$ G. N. Kakazei, T. Mewes, P. E. Wigen, P. C. Hammel, A. N. Slavin, Y. G. Pogorelov, M. D. Costa, V. O. Golub, K. Y. Guslienko, and V. Novosad, J. Nanosci. Nanotechnol. 8, 2811 (2008).

${ }^{4}$ J. Ding, M. Kostylev, and A. O. Adeyeye, Appl. Phys. Lett. 100, 062401 (2012).

${ }^{5}$ J. Podbielski, F. Giesen, and D. Grundler, Phys. Rev. Lett. 96, 167207 (2006).

${ }^{6}$ I. Neudecker, M. Kläui, K. Perzlmaier, D. Backes, L. J. Heyderman, C. A. F. Vaz, J. A. C. Bland, U. Rüdiger, and C. H. Back, Phys. Rev. Lett. 96, 057207 (2006).

${ }^{7}$ J. Topp, J. Podbielski, and D. Heitmann, Phys. Rev. B 78, 024431 (2008).

${ }^{8}$ K. L. Livesey, J. Ding, N. R. Anderson, R. E. Camley, A. O. Adeyeye, M. P. Kostylev, and S. Samarin, Phys. Rev. B 87, 064424 (2013).

${ }^{9}$ Z. K. Wang, M. H. Kuok, S. C. Ng, D. J. Lockwood, M. G. Cottam, K. Nielsch, R. B. Wehrspohn, and U. Gösele, Phys. Rev. Lett. 89, 027201 (2002).

${ }^{10}$ S. Choi, K.-S. Lee, K. Y. Guslienko, and S.-K. Kim, Phys. Rev. Lett. 98, 087205 (2007).

${ }^{11}$ M. M. Miller, G. A. Prinz, S.-F. Cheng, and S. Bounnak, Appl. Phys. Lett. 81, 2211 (2002).

${ }^{12}$ J. Ding, N. Singh, M. Kostylev, and A. O. Adeyeye, Phys. Rev. B 88, 014301 (2013).
${ }^{13}$ N. Locatelli, V. V. Naletov, J. Grollier, G. de Loubens, V. Cros, C. Deranlot, C. Ulysse, G. Faini, O. Klein, and A. Fert, Appl. Phys. Lett. 98, 062501 (2011).

${ }^{14}$ J. Ding, M. Kostylev, and A. O. Adeyeye, Appl. Phys. Lett. 100, 073114 (2012).

${ }^{15}$ O. Klein, G. de Loubens, V. V. Naletov, F. Boust, T. Guillet, H. Hurdequint, A. Leksikov, A. N. Slavin, V. S. Tiberkevich, and N. Vukadinovic, Phys. Rev. B 78, 144410 (2008).

${ }^{16}$ S. V. Nedukh, S. I. Tarapov, D. P. Belozorov, A. A. Kharchenko, V. O. Golub, I. V. Kilimchuk, O. Y. Salyuk, E. V. Tartakovskaya, S. A. Bunyaev, and G. N. Kakazei, J. Appl. Phys. 113, 17B521 (2013).

${ }^{17}$ G. N. Kakazei, P. E. Wigen, K. Y. Guslienko, V. Novosad, A. N. Slavin, V. O. Golub, N. A. Lesnik, and Y. Otani, Appl. Phys. Lett. 85, 443 (2004).

${ }^{18}$ V. Castel, J. B. Youssef, F. Boust, R. Weil, B. Pigeau, G. de Loubens, V. V. Naletov, O. Klein, and N. Vukadinovic, Phys. Rev. B 85, 184419 (2012).

${ }^{19}$ A. O. Adeyeye and N. Singh, J. Phys. D: Appl. Phys. 41, 153001 (2008).

${ }^{20}$ H. Chen, P. De Gasperis, and R. Marcelli, IEEE Trans. Magn. 29, 3013 (1993).

${ }^{21}$ E. N. Ivanov and M. Kostylev, e-print arXiv:1402.3459 [cond-mat.meshall].

${ }^{22}$ S. Tamaru, K. Yakushiji, A. Fukushima, S. Yuasa, and H. Kubota, IEEE Magn. Lett. 5, 1 (2014).

${ }^{23}$ C. Kittel, Phys. Rev. 73, 155 (1948).

${ }^{24}$ J. Ding, M. Kostylev, and A. O. Adeyeye, Phys. Rev. B 84, 054425 (2011). 